Development of longitudinal beam profile monitor based on Coherent Transition Radiation effect for CLARA accelerator

To cite this article: K. Fedorov et al 2020 JINST 15 C06008

View the article online for updates and enhancements.




XII International Symposium on Radiation from Relativistic Electrons

in Periodic Structures - RREPS-19

SEPTEMBER 16-20, 2019

Belgorod, Russian Federation

\title{
Development of longitudinal beam profile monitor based on Coherent Transition Radiation effect for CLARA accelerator
}

\author{
K. Fedorov, ${ }^{a, b, 1}$ P. Karataev, ${ }^{a}$ Y. Saveliev, ${ }^{c}$ T. Pacey, ${ }^{c}$ A. Oleinik, ${ }^{a, d}$ M. Kuimova ${ }^{b}$ \\ and A. Potylitsyn ${ }^{b}$ \\ ${ }^{a}$ John Adams Institute at Royal Holloway, University of London, \\ Egham Hill, Egham, TW20 OEX, United Kingdom \\ ${ }^{b}$ Tomsk Polytechnic University, \\ Lenina avenue, 30, 634050, Tomsk, Russia \\ ${ }^{c}$ ASTeC, STFC Daresbury Laboratory, \\ Keckwick Ln, Daresbury, Warrington WA4 4AD, United Kingdom \\ ${ }^{d}$ Laboratory of Radiation Physics, Belgorod National Research University, \\ 14 Studencheskaya Street, University Building 6, 308007 Belgorod, Russia \\ E-mail: fedorovkval@gmail.com
}

AbstRact: Coherent Transition Radiation (CTR) has widely been used for longitudinal beam profile diagnostics. Its spectral-spatial properties have been thoroughly theoretically and experimentally investigated. We have decided to develop a longitudinal beam profile diagnostics based on CTR technique to be used for interpreting the experimental results of Dielectric Wakefield Accelerator (DWA) and Coherent Cherenkov Diffraction Radiation (CChDR) experiments. In this paper we present the technical design,tuning and commissioning of the CTR longitudinal bunch profile monitor at CLARA facility.

Keywords: Cherenkov and transition radiation; Instrumentation for FEL; Interferometry; Transition radiation detectors

\footnotetext{
${ }^{1}$ Corresponding author.
} 


\section{Contents}

1 Introduction 1

2 Theoretical background 2

2.1 Reconstruction process 2

2.2 Transition radiation single electron spectrum for real parameters 2

3 Experimental setup at CLARA machine 3

4 Experimental results and discussion $\quad 6$

$\begin{array}{llr}5 & \text { Conclusion } & 8\end{array}$

\section{Introduction}

CLARA is a linear accelerator, designed to test advanced FEL concepts and capabilities for next generation X-ray FELs [1]. Electron bunches in this machine can be longitudinally compressed down to $\approx 300$ fs RMS to achieve larger currents, which is necessary to drive high-gain FEL process in an undulator magnet. Space charge forces, nonlinearities of the radio frequency fields and bunch compressors, coherent synchrotron radiation and wakefields - all have a profound influence on the time profile and internal energy distribution of the compressed bunches, so the longitudinal density profile should be accurately studied. Electro-optic methods (EO) [2] and coherent radiation techniques [3] are among the proposed options for longitudinal diagnostic at CLARA.

The principe of EO measurements is based on utilization of the field-induced birefringence in an electro-optic crystal to convert the temporal profile of a bunch into a temporal, spectral or spatial intensity modulation of a probe laser pulse. The EO techniques have the advantage of being non-destructive, preserving phase information and providing single-shot measurements. However such setup requires a properly synchronised laser system. It is also bulky and demanding on cost and man hours.

Depending on the design of the radiator and the type of radiative process, coherent radiation the technique can be destructive or non-destructive. The main advantage of the coherent radiation method is the absence of the intrinsic theoretical resolution limit on the bunch length that can be measured. The disadvantage is in difficulties with providing of single-shot diagnostic.

We have build-up a new instrument based on CTR effect $[4,5]$ to characterize longitudinal charge distribution of a single bunch on CLARA facility. We verified the methodology and compared it with another experimental method and simulations. 


\section{Theoretical background}

\subsection{Reconstruction process}

Coherent radiation appears when the radiation wavelength is comparable to, or longer than the bunch length of a charged particles beam, that interacts with the radiator. Full coherence means that the radiation fields of all $N$ electrons add constructively - in this case the radiation photon yield is proportional to the number of electrons squared. The function describing coherent emission from an electron bunch is called form-factor $F(\omega)$ and given by the Fourier transform of the longitudinal charge distribution function $\rho(z)[6]$ :

$$
F(\omega)=\left|\int_{-\infty}^{\infty} \rho(z) \exp \left(\frac{-i \omega z}{c}\right) d z\right|^{2}
$$

where $\omega$ is the angular frequency, $c$ is the speed of light. This equation can be used if the radiation is perfectly coherent in transverse direction, i.e when the transverse size of the beam is much smaller than $\gamma \lambda / 2 \pi$ product [7]. The coherent spectral radiation yield at frequency $\omega$ is [8]:

$$
\left[\frac{d W}{d \omega}\right]=\left[\frac{d W_{e}}{d \omega}\right][N+N(N-1) F(\omega)]
$$

where $\mathrm{N}$ is the number of electrons in the bunch, $d W / d \omega$ is the experimentally measured coherent spectrum; $d W_{e} / d \omega$ is theoretically calculated single electron spectrum integrated over the detector aperture. Neglecting the incoherent part of spectrum, we can say that longitudinal charge distribution is:

$$
\rho(z)=\frac{1}{\pi c} \int_{0}^{\infty} \sqrt{\frac{[d W / d \omega]}{N^{2}\left[d W_{e} / d \omega\right]}} \cos \left(\psi(\omega)-z \frac{\omega}{c}\right) d \omega
$$

where $\psi(w))$ is the minimal phase which can be derived using the Kramers-Kronig approach that takes into account phase characteristics of the measured spectrum [9]:

$$
\psi(\omega)=-\frac{2 \omega}{\pi} \int_{0}^{\infty} \frac{\ln (\sqrt{F(x)} / \sqrt{F(\omega)})}{x^{2}-\omega^{2}} d x
$$

where $x$ is integration variable in units of frequency.

Thus, the reconstruction of longitudinal charge distribution consists of 3 steps: calculation of single electron spectrum for particular experimental parameters, experimental observation of coherent spectrum emitted via interaction of a charged particles beam and the radiator, and further longitudinal distribution reconstruction with eq. (2.3) and eq. (2.4).

\subsection{Transition radiation single electron spectrum for real parameters}

TR appears when a charged particle crosses an interface between two media with different dielectric constants (for example when an electron crosses a vacuum-ideal conductor boundary). This particle induces currents changing in time at the boundary [10] and generates photons. TR has a tendency to propagate in two main directions: along the particle trajectory (forward transition radiation (FTR)) and along the direction of specular reflection from the boundary (backward transition radiation (BTR)). BTR is the one which has been widely used for different diagnostic purposes because 
it allows registration of photons emitted at significant background conditions. To calculate BTR spectral-spatial distribution and then the spectrum from a single particle we use a model described in [11], which takes into account finite TR radiator dimensions and the pre-wave zone effect. This model accounts for the following parameters: beam energy, target and detector dimensions, distance between target and detector. In these simulations it was assumed that the electron beam falls onto the radiator plane with a slant angle of $45^{\circ}$ and observed by the detector plane placed perpendicular to the angle of mirror reflection. The same setup was used in the experiments. The simulation parameters are shown in table 1 and correspond to the real experimental conditions at the CLARA machine.

Table 1. Simulation and experimental parameters.

\begin{tabular}{|l|c|}
\hline Parameters & Value \\
\hline Lorentz-factor $\gamma$ & 70 \\
Target diameter $d$ & $5 \mathrm{~cm}$ \\
Detector aperture $D$ & $4 \mathrm{~cm}$ \\
Distance between target and & \\
observation plane $L$ & $10 \mathrm{~cm}$ \\
Radiation frequency $f$ & {$[0-1.5] \mathrm{THz}$} \\
\hline
\end{tabular}

The results of simulation are shown in figure 1. Figure 1a shows the spatial distribution of horizontal polarisation component for different frequencies. The curve on figure $1 \mathrm{~b}$ is the single electron spectrum integrated over the solid angle. The reduction of spectrum intensity on the lower frequencies is caused by the finite radiator and detector dimensions. Non-uniformity of the spectrum curve is caused by the Fresnel diffraction at the radiator edges.

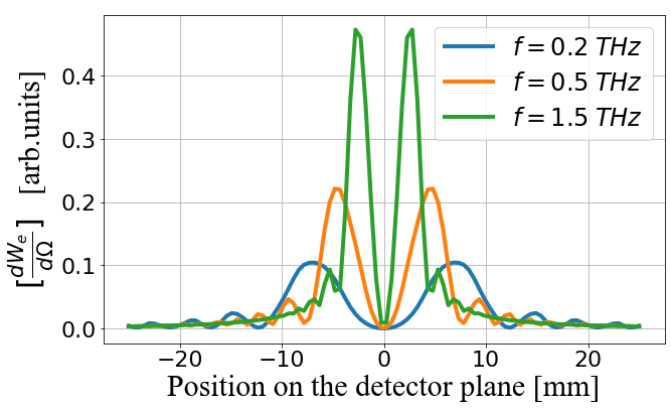

(a)

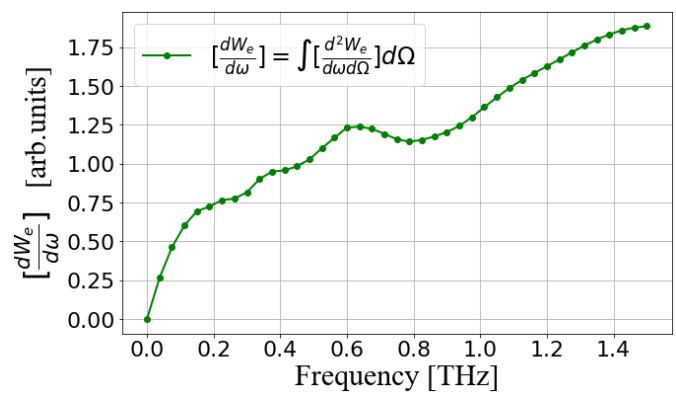

(b)

Figure 1. Simulation results of transition radiation spatial distribution (left) and single electron spectrum (right) for experimental setup installed at the CLARA facility.

\section{Experimental setup at CLARA machine}

The main part of this work was conducted at CLARA / VELA facility in Daresbury Laboratory, STFC [1]. The CLARA photoelectric gun generates a $10 \mathrm{~Hz}$ electron beam with the energy of $35 \mathrm{MeV}$ and charge of $70 \mathrm{pC}$, which is then injected into the VELA beamline. Longitudinally the 
beam is expected to be compressed from $\approx 2$ ps to 0.3 ps RMS. Longitudinal compression of the electron bunch takes place in a short dog-leg section linking CLARA and VELA beamlines and can be varied by changing the linac offcrest phase. Then the beam is delivered to the Beam Area 1 (BA1) user area, which is equipped with: large vacuum chamber accommodating a manipulation system for experimental objects, a standard diagnostics system (energy spectrometer, YAG screens, and beam position monitor) and output quartz window for observing radiation generated inside the chamber. Transverse beam size at the interaction point was typically 70-100 $\mu \mathrm{m}$ RMS.

TR target which was installed inside the chamber is a plate of polished steel, specially manufactured for this experiment with a radius of $2 \mathrm{~cm}$ and thickness of $2 \mathrm{~mm}$. It was mounted on a movable holder and located on the platform with 2 degrees of translational freedom - vertical and horizontal as shown in figure 2. BTR (red arrow) induced by an electron (yellow arrow) hitting

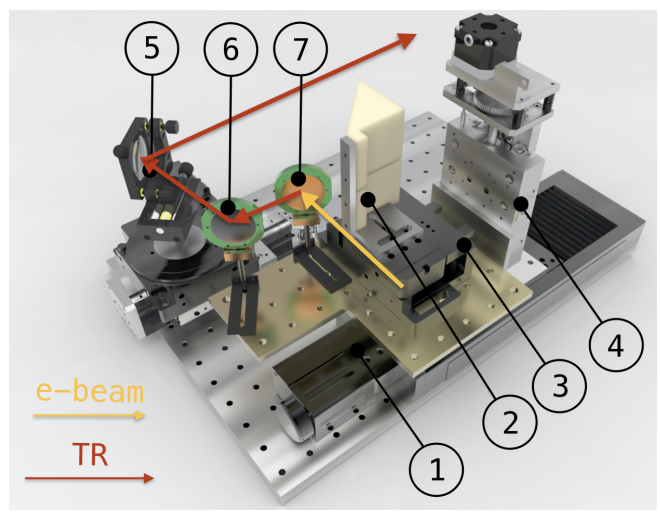

(a)

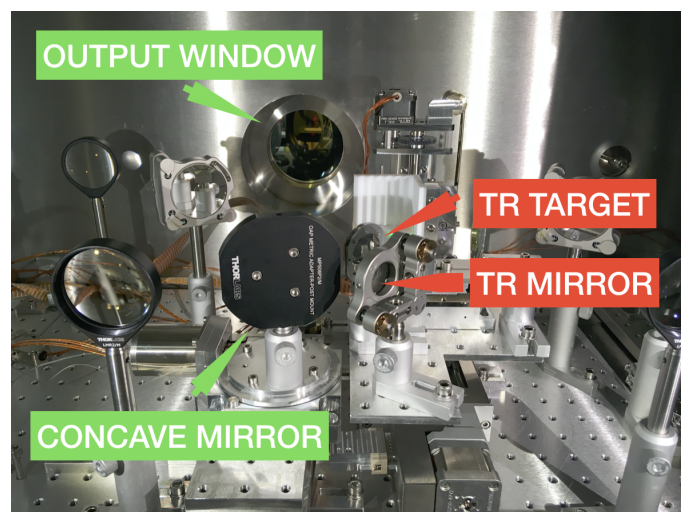

(b)

Figure 2. (a) Setup inside vacuum chamber: 1 - horizontal positioning stage, 2 - ChDR target (method with non-invasive coherent Cherenkov diffraction radiation described in [19, 20], 3 - tip-tilt stage, 4 - vertical positioning stage, 5 - concave mirror, 6 - TR steer mirror, TR radiator; (b) photo of the experimental setup inside a vacuum chamber.

the radiator, then radiation is reflected by a steer mirror, then collimated by an off-axis parabolic mirror (it was installed at $101.6 \mathrm{~mm}$ from the CTR target which is its focal length) and delivered to the detection system via output quartz window. Further, BTR was delivered into a Martin-Puplett interferometer and a system of motorized mirrors.

The Martin-Puplett interferometer [12] is one of the most efficient instruments to measure a radiation spectrum in the near millimeter range. The main advantage of it is the design with a polarisation splitter, which provides greater efficiency of the device, as well as the ability to eliminate the influence of current fluctuations in the accelerator using two detectors (PD1 and PD2 in figure 3). The signal from these detectors is anticorrelated relative to each other. Therefore, if we divide the difference between these two signals by their sum, we will get a normalized interferogram $\delta(x)$ :

$$
\delta(x)=\frac{I_{\mathrm{PD} 1}-I_{\mathrm{PD} 2}}{I_{\mathrm{PD} 1}+I_{\mathrm{PD} 2}}
$$

In this case, it becomes absolutely necessary to ensure the highest quality alignment of all elements of the interferometer. The alignment was performed using a test source and a Terasence camera operating at $10 \mathrm{GHz}-1 \mathrm{THz}$ [13]. 


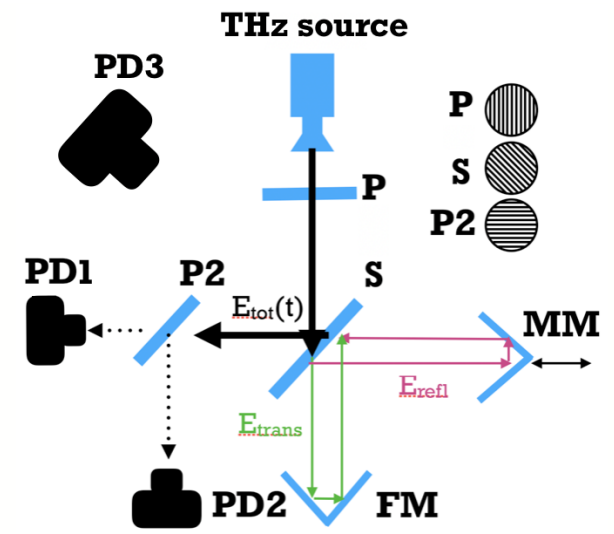

(a)

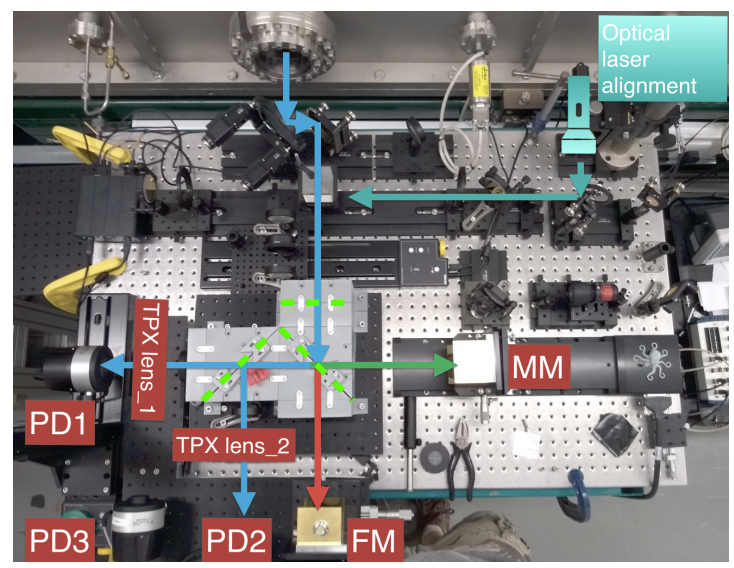

(b)

Figure 3. Setup outside vacuum chamber. (a) Schematic view on MPI: P, P2 - polarisers, S - polarisation spliter, MM - movable roof mirror, FM - fixed roof mirror, PD1 and PD2 - pyroelectric detectors (Gentec THz51); additional pyroelectric detector PD3 was used to monitor the noise in the environment and provide a shot-to-shot background correction. The wire grids transmit polarisation perpendicular to the wire orientation, therefore the field after the polariser $\mathrm{P}$ is horizontally polarised; (b) photo of spectroscopy setup
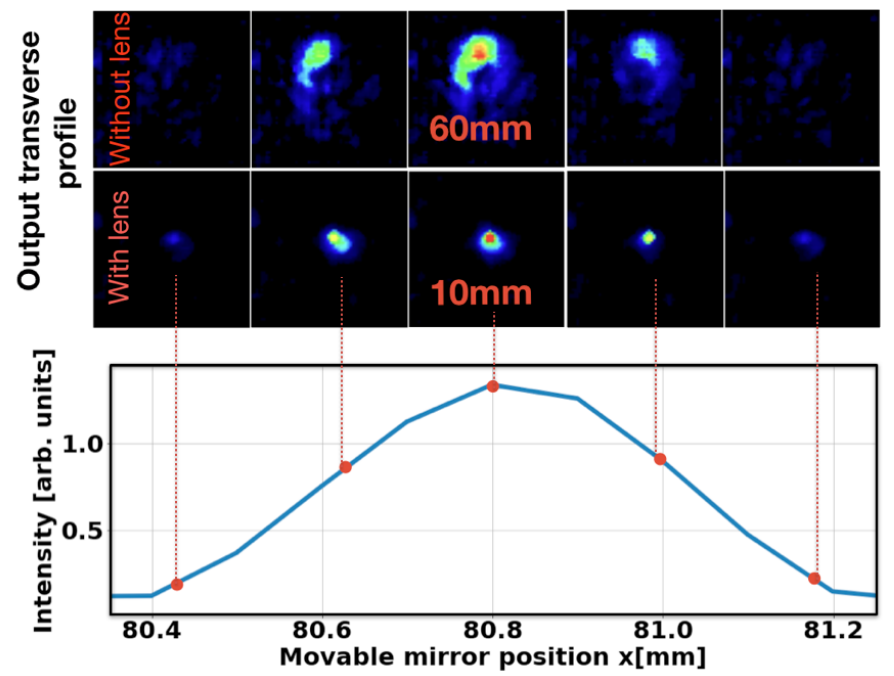

Figure 4. Visualization and alignment using $\mathrm{THz}$ camera and $\mathrm{CW} \mathrm{THz}$ source operating at $170 \mathrm{GHz}$ : above - images of transverse profile for different movable mirror positions with and without TPX lenses (Tydex LLC.) required for choosing the optimal detector displacement; below - interference pattern corresponding to the images above.

Figure 4 shows the example of using visualization and alignment with the $\mathrm{THz}$ camera. Test $\mathrm{CW} \mathrm{THz}$ source operating at $170 \mathrm{GHz}$ frequency was installed inside of a vacuum chamber and simulated the TR radiator. THz radiation from the test source propagated through entire optical system including MPI interferometer and then it falls onto THz camera (Terasence, [13]). The top figure is the images of a signal from the test constant-wave $\mathrm{THz}$ source measured by the Terasence camera placed in detector plane with and without the lens. This images correspond to 
the interference pattern shown below. This approach allowed us to choose optimal focal lengths of lenses and the detector displacement. By observing constructive and destructive interference patterns, we can adjust the direction of photons through the interferometer.

During TR scan the data acquisition system was synchronized with the accelerator and recorded 50 samples from each pyrodetector for one step of the moving mirror. Then this data were averaged and separately stored for off-line data processing. The averaging error was also recorded and used for post-processing.

\section{Experimental results and discussion}

The results of CTR interferometry are shown in figure 5. Figure 5a demonstrates anticorrelated signals from PD1 and PD2 (after using apodization procedure with the Blackman window [14]), in figure $5 \mathrm{~b}$ one may see the normalized interferogram, $\delta(x)$, calculated using eq. (3.1).

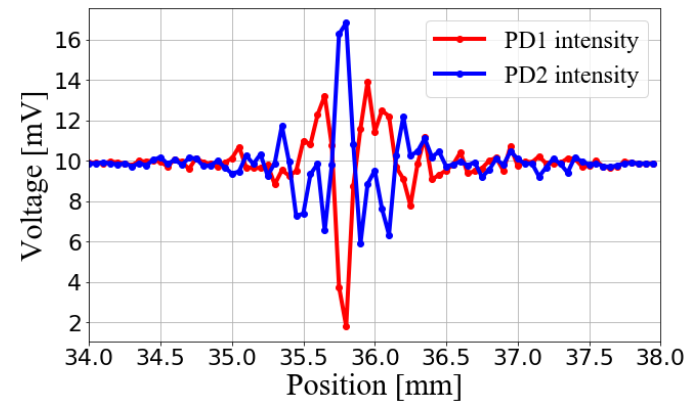

(a)

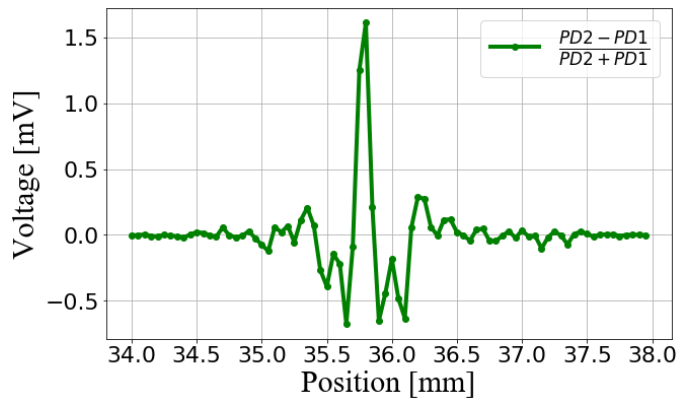

(b)

Figure 5. (a) Interferograms measured from two different detectors PD1 and PD2; (b) normalized interfero$\operatorname{gram} \delta(x)$.

The CTR spectrum in figure 6a (green line) is calculated using the Fourier transform of $\delta(x)$. At lower frequencies both spectra decrease in intensity due to diffraction effects in the radiation delivery system. At higher frequencies spectra depends on the longitudinal bunch form-factor. For frequencies more than $800 \mathrm{GHz}$, the decisive factor is low signal-to-noise ratio of pyroelectric detector. Nevertheless, frequencies below $200 \mathrm{GHz}$ and above $800 \mathrm{GHz}$ need to be extrapolated for further analysis. Also in figure 6a humid air absorption lines are shown, which are theoretical data from [15]. To provide normalization and exclude the influence of water lines in the air we used Gaussian filter in which $\sigma$ corresponds to the spectrum resolution of the interferometer ( $f=\frac{c}{2 L}=37.5 \mathrm{GHz}$, where $\mathrm{c}$ is the speed of light, $\mathrm{L}$ is the length of interferometer). In figure $6 \mathrm{~b}$ CTR spectrum normalized by absorption spectrum of water in humid air is shown.

As was mentioned above, the form-factor is a product of division of coherent spectrum (figure $6 \mathrm{~b}$ ) by a single electron spectrum presented in figure 1b. Extrapolated form-factor and minimal phase calculated with eq. (2.4) are shown in figure 7a. Figure $7 \mathrm{~b}$ shows the final result of longitudinal profile reconstruction. FWHM (Full Width at Half Maximum) duration of the bunch core part for this particular regime is $\approx 0.648( \pm 0.027$ regression error $)$ ps. At these particular machine settings, the bunch was slightly overcompressed in the dog-leg that leads to the formation of 


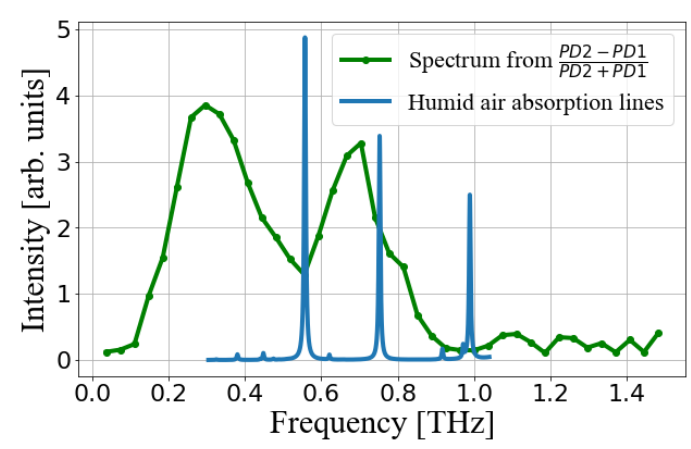

(a)

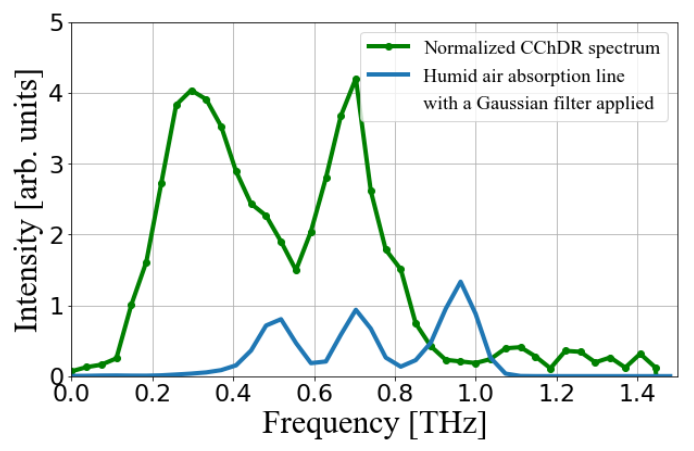

(b)

Figure 6. (a) Spectrum obtained by using FFT of normalized interferogram $\delta(x)$ and humid air absorption lines from [15]; (b) Humid air absorption lines after applying Gaussian filter for corresponding frequencies and CTR spectrum normalized on humid air lines.

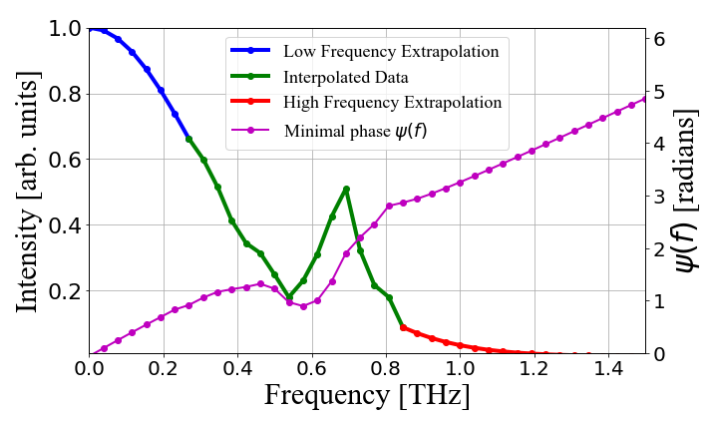

(a)

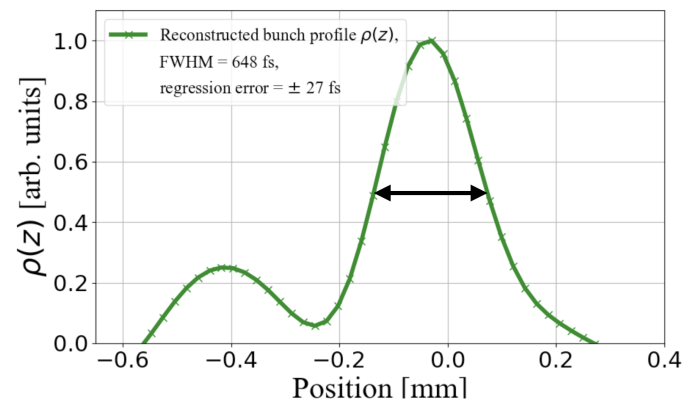

(b)

Figure 7. (a) Extrapolated form-factor $F(f)$ and minimal phase $\psi(f)$, (b) Result of longitudinal profile reconstruction using eq. (2.2). Extrapolation to lower and higher frequencies performed by a Gaussian and exponential curve, following the prescriptions in [16].

the current peak at the head and lower current tail. These settings also increase the bunch-to-bunch jitter, therefore, the shape of the tail should be considered as average over multiple shots.

The aim of the experimental work was not only to show the longitudinal bunch profile, but also to observe the sensitivity of the bunch core length to small variation of bunch compression parameters. Figure 8 shows the dependence of the bunch length on the linac phase obtained by CTR, ELEGANT code prediction and induced energy modulation technique [17, 18]. It can be seen that the developed instrument based on the CTR effect demonstrates a good consistency with other technique and expectations. The slight difference in these dependencies is caused by a lack of higher frequency signal, which can be improved by using more sensitive detectors, such as, for example, Schottky diode detectors. 


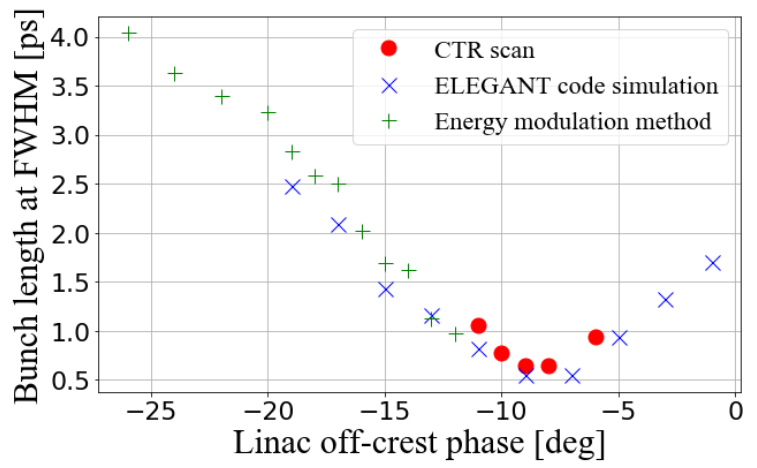

Figure 8. Comparison of bunch length versus linac offcrest phase dependencies for ELEGANT code prediction, Dielectric Wakefield Acceleration method and diagnostic with coherent transition radiation.

\section{Conclusion}

We demonstrated a complete process of longitudinal bunch profile diagnostic using coherent transition radiation at the CLARA facility. With precise alignment, the transition radiation from a steel target was easily detectable. In combination with the Martin-Puplett interferometer, it gives a quasicontinuous spectrum. The obtained results are in good agreement with simulations performed for the CLARA machine and with other diagnostic method. This setup was already successfully applied for interpritation of DWA [18] and CChDR [19, 20] experiments as a gauge method of diagnostic. The complete cycle of bunch length measurement (including the measurement of interferogram, FFT, single spectrum normalization, and Kramers-Kronig bunch profile reconstruction) could take up to 15 minutes with current experimental procedure and this can be reduced to one minute by using detectors with a higher signal-to-noise ratio, for example, quazi-optical Schottky detectors, and automating the analysis.

\section{References}

[1] P.A. McIntosh et al., The VELA and CLARA Test Facilities at Daresbury Laboratory, in Proc. 28th Linear Accelerator Conf. (LINAC'16), East Lansing, MI, U.S.A., September 2016, pp. 734 [DOI].

[2] D. Walsh et al., The time resolved measurement of ultrashort terahertz-band electric fields without an ultrashort probe, Appl. Phys. Lett. 106 (2014) 181109.

[3] O. Grimm and P. Schmuser, Principles of longitudinal beam diagnostics with coherent radiation, TESLA-FEL Report (2006) [https://www.osti.gov/etdeweb/servlets/purl/20851651].

[4] I. Nozawa et al., Measurement of $<20 f$ s bunch length using coherent transition radiation, Phys. Rev. ST Accel. Beams 17 (2014) 072803.

[5] X. Su et al., Monitoring of electron bunch length by using Terahertz coherent transition radiation, Nucl. Instrum. Meth. B 402 (2017) 157.

[6] J.S. Nodvick and D.S. Saxon, Suppression of Coherent Radiation by Electrons in a Synchrotron, Phys. Rev. 96 (1954) 180.

[7] A.Potylitsyn et al., Diffraction Radiation from Relativistic Particles, Springer Tracts Mod. Phys. 239 (2010). 
[8] Y.Shibata et al., Observation of coherent transition radiation at millimeter and submillimeter wavelengths, Phys. Rev. A 45 (1992) 340.

[9] R. Lai and A.J. Sievers, Phase problem associated with the determination of the longitudinal shape of a charged particle bunch from its coherent far-ir spectrum, Phys. Rev. E 52 (1995) 4576.

[10] M. Ter-Mikaelyan, High Energy Electromagnetic Processes in Condensed Media, Wiley-Interscience, New York (1972) [CERN Document Server].

[11] P. Karataev, Pre-wave zone effect in transition and diffraction radiation: Problems and solutions, Phys. Lett. A 345 (2005) 428.

[12] D. Martin and E. Puplett Polarised interferometric spectrometry for the millimetre and submillimetre spectrum, Infrared Phys. 10 (1970) 105.

[13] Terasence THz camera datasheet, http://terasense.com/products/sub-thz-imaging-cameras/.

[14] R. Bretzlaff and T. Bahder, Apodization effects in Fourier transform infrared difference spectra, Rev. Phys. Appl. 21 (1986) 833.

[15] D. Slocum et al., Atmospheric absorption of terahertz radiation and water vapor continuum effects, J. Quant. Spectrosc. Radiat. Trans. 127 (2013) 49.

[16] M. Micheler et al., Longitudinal beam profile monitor at ctf3 based on co- herent diffraction radiation, J. Phys. Conf. Ser. 236 (2010) 012021.

[17] S. Antipov et al., Experimental Observation of Energy Modulation in Electron Beams Passing Through Terahertz Dielectric Wakefield Structures, Phys. Rev. Lett. 108 (2012) 144801 [arXiv: 1111.7291].

[18] T.H. Pacey et al., Continuously tunable narrow-band terahertz generation with a dielectric lined waveguide driven by short electron bunches, Phys. Rev. Accel. Beams 22 (2019) 091302.

[19] K. Fedorov et al., Experimental observationof submillimeter coherent Cherenkov radiation at CLARA facility, presented at The 8th Int. Beam Instrumentation Conf. (IBIC'19), Malmö, Sweden, September 2019, paper TUCO02 [http://ibic2019.vrws.de/papers/tuco02.pdf].

[20] A. Curcio et al., Noninvasive bunch length measurements exploiting Cherenkov diffraction radiation, Phys. Rev. Accel. Beams 23 (2020) 022802. 